\title{
The Limits of Extended Kalman Filtering for Pulse Train Deinterleaving
}

\author{
Tanya Conroy and John B. Moore, Fellow, IEEE
}

\begin{abstract}
Some signals, such as in radar systems, communication systems, and neural systems, are transmitted as periodic pulse trains. If more than one pulse train is transmitted over the same communication channel, a challenge is to separate them for source identification at the receiver. This is known as pulse train deinterleaving and is clearly a fundamental problem in the study of discrete-event systems. Frequently, the only relevant information at the receiver is the time of arrival (TOA) data, which is usually contaminated by jitter noise. Perhaps there are also missing or overlapping pulses.

In this paper, we present an approach for deinterleaving pulse trains and estimating their periods using an extended Kalman filter (EKF). A naive application of EKF theory is not attractive because of discontinuities in the signal model. Here, a form of smoothing of the discontinuities is proposed so that the EKF approach becomes attractive. The advantage of this EKF approach is that it is less computationally expensive than most previously proposed methods, which are of order $N^{2}$, where $N$ is the number of pulses being processed. The computation required here is of order $N$. The method proposed appears to give useful results for up to seven or so pulse trains, particularly when there is some a priori information on the pulse frequencies, which can be obtained using computations of order $N \log N$.
\end{abstract}

Index Terms -Deinterleaving, extended Kalman filtering.

\section{INTRODUCTION}

$\mathbf{S}$ OME SIGNALS are transmitted as periodic pulse trains. Consider a situation where pulses from a number of different sources are being transmitted over a single communication channel. This leads to a series of pulse trains that are said to be interleaved. The process of pulse train deinterleaving is separating these pulses into the original trains. In order to do this, use is made of the fact that the different trains have different characteristics, such as period of pulse emission, phase, and pulse amplitude. Here, we restrict our attention to the case where only time of arrival (TOA) data for the received pulses is available or relevant. Deinterleaving is fundamentally a difficult problem, even in the ideal case with no jitter noise, missing or overlapping pulses, or period variations, but it is important that there be some robustness in the nonideal case. Pulse train deinterleaving is used in radar detection [1] and could potentially be studied in the areas of computer communications and neural systems.

Manuscript received July 10, 1997; revised May 26, 1998. The associate editor coordinating the review of this paper and approving it for publication was Prof. Peter C. Doerschuk.

The authors are with the Department of Systems Engineering, Research School of Information Sciences and Engineering, Australian National University, Canberra, Australia.

Publisher Item Identifier S 1053-587X(98)08817-5.
Previously proposed techniques for pulse train deinterleaving include sequential search [2] and histogramming [2], [3]. These techniques work well when the interleaved signal is received in low noise. Another approach is to formulate the problem as a stochastic discrete-time dynamic linear model [4]. Here, the deinterleaving methods used are forward dynamic programming with fixed look-ahead and a probabilistic teacher.

A problem with all the above methods is that they are computationally expensive, typically of order $N^{2}$ or higher, where $N$ is the number of pulses being processed. Optimal processing involves a full tree search, requiring computational effort of order $M^{N}$ for $M$ pulse trains. One method uses fast Fourier transform techniques to determine the number of pulse trains present in an interleaved signal and estimate their periods (their spectra) without actually deinterleaving them [5]. The computation required here is of order $N \log N$.

In this paper, the signal model from [4] is modified by a smoothing of its inherent discontinuities so that the deinterleaving task can be performed using the extended Kalman filter with computational effort of order $N$. It is assumed that the pulse trains are periodic and that the number of sources is finite and known. It is also desirable that the processing exploit a priori information as from spectra determined in [5].

This paper is structured as follows. In Section II, the problem is formulated in terms of a state space signal model, including a version with smooth nonlinearities. In Section III, the extended Kalman filter is presented. In Section IV, simulation examples are presented, and in Section V, some robustness issues are examined.

\section{Signal Model-A $\mu$ Parameterization}

Consider $M$ periodic pulse train sources. Let $T^{(i)}$ and $t_{0}^{(i)}$ denote, respectively, the period and initial phase of the $i$ th source. The received interleaved signal consists of the superposition of the $M$ pulse trains produced by these sources. Let $t_{1}, t_{2}, \cdots, t_{N}$ denote the times of arrival of $N$ consecutive pulses. The problem is as follows.

Problem: Given the pulses $t_{1}, \cdots, t_{N}$ and the number of sources present $M$, determine which source produced each pulse and estimate the periods $T^{(i)}$ and phases $t_{0}^{(i)}$ of each pulse train for $i=1,2, \cdots, M$.

This is a complex problem, as shown in Fig. 1. Here, pulses from interleaved pulse trains are shown with no information other than TOA data to identify their source. It is possible to deinterleave the two source train by eye, but this task quickly becomes impossible as more sources are added. 


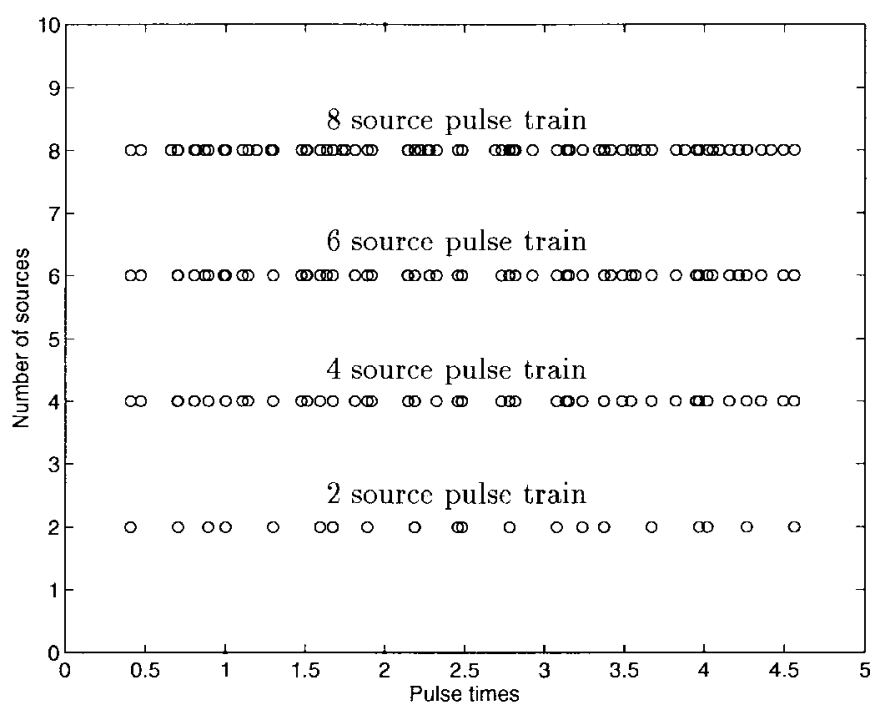

Fig. 1. Interleaved pulse trains.

The signal can be described by a discrete-state, discrete-time model where the index is pulse number and not time. That is, the model updates not at some discrete-time period but with each pulse received. We consider the model proposed in [4].

$$
\begin{aligned}
& x_{k+1}=\left[\begin{array}{cc}
I & 0 \\
X_{k}^{\operatorname{diag}} & I
\end{array}\right] x_{k}, x_{0} \\
& y_{k+1}=\left[\begin{array}{ll}
X_{k}^{\prime} & X_{k}^{\prime}
\end{array}\right] x_{k}+\omega_{k}
\end{aligned}
$$

where

$k \quad$ pulse number;

$x_{k} \quad$ state variable at $k$;

$X_{k}$ source indicator vector;

$y_{k} \quad$ received signal at $k$.

Here, $\omega_{k}$ is zero-mean white Gaussian noise (WGN) on the received signal with known variance $\sigma_{\omega}^{2}$, which is termed the jitter noise.

The state variable takes the form

$$
x_{k}^{\prime}=\left[T_{k}^{(1)}, \cdots, T_{k}^{(M)}, t_{k}^{(1)}, \cdots, t_{k}^{(M)}\right]
$$

where there are $M$ pulse train sources, $T_{k}^{(i)}$ is the period of train $i$, and $t_{k}^{(i)}$ is the time of arrival of the most recent pulse in train $i$ at pulse number $k$. Clearly, $t_{k}^{(i)} \leq y_{k}$.

The source indicator vector $X_{k} \in\left\{e_{1}, e_{2}, \cdots, e_{M}\right\}$, where $e_{i}$ are unit column vectors in $R^{N}$ with the 1 in the $i$ th position, indicates the source-generated pulse $k$. If $X_{k}=e_{i}$, then the $i$ th source is active at the $k$ th pulse, and the value of $t_{k}^{(i)}$ for that train is increased by the period of the train, whereas all others remain constant. It is assumed in the first instance that only one source is active when a pulse is received. This eliminates the case when the sources are integer multiples, for example.

A key observation in this paper is that the source indicator vector $X_{k}$ can be expressed in terms of the state as

$$
X_{k}\left(x_{k}\right)=e_{i^{*}}
$$

where $i^{*}$ is the $\arg \min _{i}\left\{\cdots,\left(T_{k}^{(i)}+t_{k}^{(i)}\right), \cdots\right\}$. This indicator vector is discontinuous in $x_{k}$.
The signal model (1) can be fleshed out as a nonlinear state space model

$$
\begin{aligned}
x_{k+1} & =f_{k}\left(x_{k}\right), x_{0} \\
y_{k+1} & =h_{k}\left(x_{k}\right)+\omega_{k}
\end{aligned}
$$

where

$$
\begin{aligned}
& f_{k}\left(x_{k}\right)=\left[\begin{array}{cc}
I & 0 \\
X_{k}^{\operatorname{diag}}\left(x_{k}\right) & I
\end{array}\right] x_{k} \\
& h_{k}\left(x_{k}\right)=\left[\begin{array}{ll}
X_{k}^{\prime}\left(x_{k}\right) & X_{k}^{\prime}\left(x_{k}\right)
\end{array}\right] x_{k} .
\end{aligned}
$$

Notice that this signal model has discontinuous nonlinearities $f_{k}(\cdot), h_{k}(\cdot)$ and, thus, cannot be used without modification to derive an extended Kalman filter (EKF).

\section{A. A Smooth Approximation of $X_{k}\left(x_{k}\right)$, Denoted $X_{k}^{\mu}\left(x_{k}\right)$}

In order to use an extended Kalman filter for deinterleaving, we propose to approximate the source indicator vector function given in (3) by a smooth function as

$$
\begin{aligned}
X_{k}^{\mu} & =\left[\left(X_{k}^{\mu}\right)^{1}, \cdots,\left(X_{k}^{\mu}\right)^{M}\right]^{\prime} \\
\left(X_{k}^{\mu}\right)^{i} & =\frac{\left(\tilde{t}_{k}^{(i)}+T_{k}^{(i)}\right)^{-\mu}}{\sum_{j}\left(\tilde{t}_{k}^{(j)}+T_{k}^{(j)}\right)^{-\mu}} \\
\tilde{t}_{k}^{(i)} & =t_{k}^{(i)}-\min _{j}\left(t_{k}^{(j)}\right) .
\end{aligned}
$$

$\tilde{t}_{k}^{(i)}$ is simply a scaling of pulse time of arrival $t_{k}^{(i)}$ to the order of the pulse train period $T_{k}^{(i)}$ for all $k$. This is done to avoid ill conditioning of $X_{k}^{\mu}$ as $k$ increases due to $t_{k}^{(i)}$ increasing with $k$, whereas $T_{k}^{(i)}$ remains constant. If this was not done, then for high $k, X_{k}^{\mu}$ would award the $(k+1)$ th pulse to the train that was awarded the $k$ th pulse, with no consideration given to the pulse train periods.

Notice that in (5)

$$
\begin{aligned}
\lim _{\mu \rightarrow \infty} X_{k}^{\mu} & =X_{k} \\
\underline{1}^{\prime} X_{k}^{\mu} & =1,\left(X_{k}^{\mu}\right)^{i} \geq 0 \quad \text { for } \quad i=1,2, \cdots, M
\end{aligned}
$$

where $\underline{1}^{\prime}=[1,1, \cdots, 1]$. That is, $X_{k}^{\mu}$ belongs to a simplex denoted $\Delta^{M-1}$ with vertices $e_{1}, e_{2}, \cdots, e_{M}$. We can think of $X_{k}^{\mu}$ as being a vector of probabilities, with $\left(X_{k}^{\mu}\right)^{i}$ as the probability of source $i$ being active. This interpretation makes good sense when dealing with estimates of the states, which are denoted $\hat{x}_{k}$, leading to estimates of $X_{k}$ or $X_{k}^{\mu}$, which are denoted $\hat{X}_{k}, \hat{X}_{k}^{\mu}$, which also belong to $\Delta^{M-1}$.

The source indicator vector from (5) for three sources is illustrated in Fig. 2, which depicts the simplex $\Delta^{2}$ with vertices $e_{1}, e_{2}, e_{3}$. Note that the axes are labeled according to the notation used in (3). This illustrates the discontinuous nature of $X_{k}$. It can only take the three values that mark the vertices of the simplex, whereas $X_{k}^{\mu}$ can take any value on the simplex. Here, source 1 is active; therefore, $X_{k}^{\mu}$ lies closest to $X_{k}=e_{1}$.

The smoothed signal model is now

$$
\begin{aligned}
x_{k+1} & =f_{k}^{\mu}\left(x_{k}\right), x_{0} \\
y_{k+1} & =h_{k}^{\mu}\left(x_{k}\right)+\omega_{k}
\end{aligned}
$$




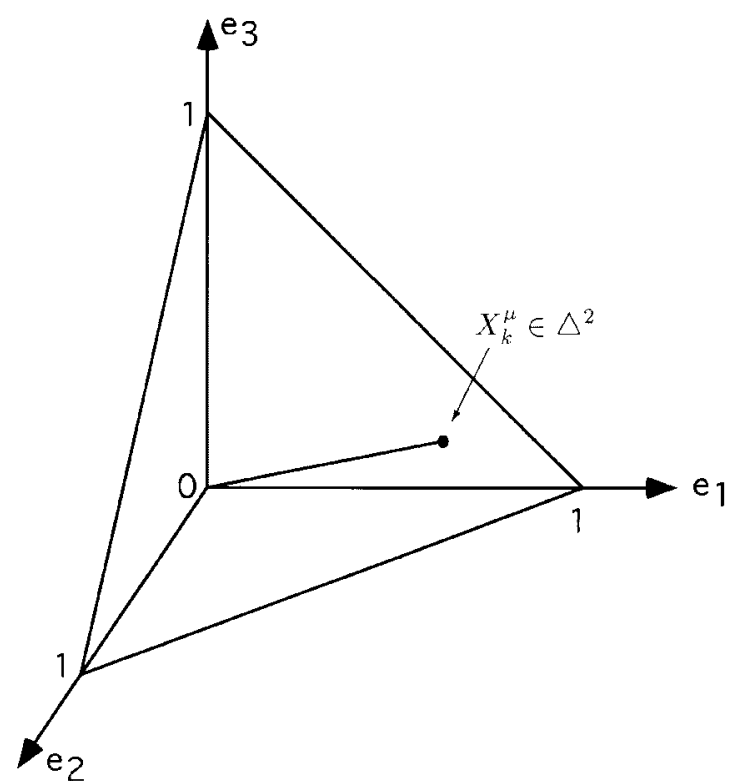

Fig. 2. Illustration of source indicator vector and simplex $\Delta^{2}$.

where

$$
\begin{aligned}
& f_{k}^{\mu}\left(x_{k}\right)=\left[\begin{array}{cc}
I & 0 \\
X_{k}^{\mu \operatorname{diag}}\left(x_{k}\right) & I
\end{array}\right] x_{k} \\
& h_{k}^{\mu}\left(x_{k}\right)=\left[\begin{array}{ll}
X_{k}^{\mu \prime}\left(x_{k}\right) & X_{k}^{\mu \prime}\left(x_{k}\right)
\end{array}\right] x_{k} .
\end{aligned}
$$

Calculation of the Smoothing Coefficient $\mu$ : It is desirable that the value of $\mu$ increase as the system states become better known. That is, when there is high certainty about the states, estimates $\hat{X}_{k}^{\mu}$ should be placed toward the simplex vertex indicated by $X_{k}$; therefore, $\mu$ should be large. This leads to an empirical calculation of $\mu$ using the change in the estimated periods over a set number of pulses.

To avoid ill conditioning, $\mu$ is constrained between fixed lower and upper values. An equation for $\mu$ is set up in such a way that when the estimated periods are fluctuating, $\mu$ is at its lower value, and once the estimated periods have reached their steady-state values, $\mu$ takes its upper value. This is done by observing the variation in the periods over, say, 50 pulses. If the maximum variation is of the order of five times the known noise variance, i.e., $5 O\left(\sigma_{\omega}^{2}\right)$, then steady state has been reached. If the maximum variation is more than five times above this $\left[25 O\left(\sigma_{\omega}^{2}\right)\right]$, then the periods are still fluctuating. An intermediate value for $\mu$ is used when the maximum variation is between these values.

\section{The Extended Kalman Filter}

In order to construct an extended Kalman filter from the smoothed signal model given in (6) denoted $\operatorname{EKF}(\mu)$, linearized versions of $f_{k}^{\mu}$ and $h_{k}^{\mu}$ (7) are needed. They are

$$
\begin{aligned}
F_{k}^{\mu} & =\left[\begin{array}{cc}
I & 0 \\
X_{k}^{\mu \operatorname{diag}}+S_{F T}^{\mu} & I+S_{F t}^{\mu}
\end{array}\right] \\
H_{k}^{\mu} & =\left[\begin{array}{ll}
X_{k}^{\mu \prime}+S_{H T}^{\mu} & X_{k}^{\mu \prime}+S_{H t}^{\mu}
\end{array}\right] .
\end{aligned}
$$

The values of $S_{H T / t}^{\mu}$ in $H_{k}^{\mu}$ are called the sensitivity functions of $H_{k}^{\mu}$. They are a measure of how much
$\left[X_{k}^{\mu \prime}\left(x_{k}\right) \quad X_{k}^{\mu \prime}\left(x_{k}\right)\right]$ varies with small changes in $T_{k}$ and $t_{k}$, respectively. This is similar for $S_{F T / t}^{\mu}$ in $F_{k}^{\mu}$.

Let us now define two cases for the $\operatorname{EKF}(\mu)$ deinterleaver. Case $1[\operatorname{EKF}(\infty)]$ is where $\mu=\infty$; therefore, $X_{k}^{\mu}=X_{k}$ (3). Case 2 is where $\mu$ is allowed to take a range of values, and $X_{k}^{\mu}$ is defined in (5). Case 1 is not appropriate for use in an EKF deinterleaver as $X_{k}$ is discontinuous and, therefore, cannot be linearized, except by ignoring aspects of the discontinuities, that is, by assuming that $X_{k}$ does not vary with $T_{k}$ or $t_{k}$ or, equivalently, setting $S_{F T / t}^{\mu}$ and $S_{H T / t}^{\mu}$ to zero.

For case 2 , the equations for the sensitivity functions of $F_{k}^{\mu}$ are

$$
S_{F T}^{\mu}=\left\{\frac{\mu\left(A_{k}^{(i)}\right)^{-(\mu+1)}\left[\sum_{l}\left(A_{k}^{(l)}\right)^{-\mu}-\left(A_{k}^{(i)}\right)^{-\mu}\right]}{\left[\sum_{l}\left(A_{k}^{(l)}\right)^{-\mu}\right]^{2}} T_{k}^{(i)}\right\}
$$

$$
S_{F t}^{\mu(i, i)}=S_{F T}^{\mu(i, i)} \hat{t}_{k}^{(i)}
$$

where $A_{k}^{(a)}=\widetilde{t}_{k}^{(a)}+T_{k}^{(a)}$, and the equations for the sensitivity functions of $H_{k}^{\mu}$ are

$$
\begin{aligned}
S_{H T}^{\mu(i)}= & \sum_{j}\left[\frac{-\mu\left(A_{k}^{(i)}\right)^{-(\mu+1)}\left(A_{k}^{(j)}\right)^{-\mu}\left(t_{k}^{(j)}+T_{k}^{(j)}\right)}{\left[\sum_{l}\left(A_{k}^{(l)}\right)^{-\mu}\right]^{2}}\right] \\
& +\frac{\mu\left(A_{k}^{(i)}\right)^{-(\mu+1)}\left(t_{k}^{(i)}+T_{k}^{(i)}\right)}{\sum_{l}\left(A_{k}^{(l)}\right)^{-\mu}} \\
S_{H t}^{\mu(i)}= & S_{H T}^{\mu(i)} \hat{t}_{k}^{(i)}
\end{aligned}
$$

where $A_{k}^{(a)}=\tilde{t}_{k}^{(a)}+T_{k}^{(a)}$.

The introduction of $\hat{t}_{k}^{(i)}$ in (9b) and (10b) is an approximation to remove a discontinuity introduced in the normalization procedure from (5). The exact equation for $S_{F t}^{\mu(i, i)}$ is

$$
S_{F t}^{\mu(i, i)}= \begin{cases}S_{F T}^{\mu(i, i)}, & \text { if } t_{k}^{i} \neq \min _{j}\left(t_{k}^{(j)}\right) \\ 0, & \text { otherwise. }\end{cases}
$$

Since $\tilde{t}_{k}^{(i)}=t_{k}^{(i)}-\min _{j}\left(t_{k}^{(j)}\right)$ from (5), $\tilde{t}_{k}^{(i)}$ varies between zero and some number in the order of the train period. Therefore, $S_{F t}^{\mu(i, i)}$ no longer takes exactly the values of zero or $S_{F T}^{\mu(i, i)}$ but varies so that the discontinuity is smoothed. This is similar for $S_{H t}^{\mu(i)}$. Since the sensitivity terms are only used to give a feel for what is occurring (see below), this approximation is acceptable.

The sensitivity terms are approximately of the order of $X_{k}^{\mu}$, except when a train is about to produce a pulse. Then, the sensitivity terms $S_{F T / t}^{\mu}$ become so large that they swamp the other terms in $F_{k}^{\mu}$ while $S_{H T / t}^{\mu}$ goes negative. It is important that this information be reflected in $F_{k}^{\mu}$ and $H_{k}^{\mu}$ but not dominate the other terms. For this reason, $S_{F T / t}^{\mu}$ and $S_{H T / t}^{\mu}$, 
although included in the calculations for $F_{k}^{\mu}$ and $H_{k}^{\mu}$, are limited in magnitude.

\section{A. The Riccati Equation}

The Riccati equation for the extended Kalman filter is

$$
\begin{aligned}
K_{k}^{\mu} & =P_{k / k-1} H_{k}^{\mu}\left(H_{k}^{\mu \prime} P_{k / k-1} H_{k}^{\mu}+R_{k}\right)^{-1} \\
P_{k+1 / k} & =F_{k}^{\mu}\left(P_{k / k-1}-K_{k}^{\mu} H_{k}^{\mu \prime \prime} P_{k / k-1}\right) F_{k}^{\mu \prime}+Q_{k}
\end{aligned}
$$

where

$K_{k}^{\mu} \quad$ Kalman gain;

$P_{k / k-1}$ error covariance at $k$, given measurements to $k-1$;

$R_{k} \quad$ covariance of the noise on the measurement;

$Q_{k} \quad$ covariance of the noise caused by smoothing.

The initialization here is provided by $P_{0 /-1}=P_{0}$.

Now

$$
\begin{aligned}
& R_{k}=\left[\begin{array}{ll}
T_{k}^{\prime} & t_{k}^{\prime}
\end{array}\right]\left[\begin{array}{cc}
\Sigma & \Sigma \\
\Sigma & \Sigma
\end{array}\right]\left[\begin{array}{l}
T_{k} \\
t_{k}
\end{array}\right]+r_{k}^{\mu} \\
& Q_{k}=T_{k}^{\operatorname{diag}}(\Sigma) T_{k}^{\operatorname{diag}}+q_{k}^{\mu} I
\end{aligned}
$$

where $\Sigma=X_{k}^{\mu \operatorname{diag}}-X_{k}^{\mu} X_{k}^{\mu \prime}$.

The constants $r_{k}^{\mu}$ and $q_{k}^{\mu}$ are added to the lower bound noise covariance equations to represent model errors and, therefore, to enhance robustness. For the case $2 \operatorname{EKF}(\mu)$ deinterleaver, they are tied to the value of $\mu$ and, hence, the certainty in the system. As $\mu$ increases, $r_{k}^{\mu}, q_{k}^{\mu}$ decrease. See Section II-A1 for a description of the calculation of $\mu$. For the case 1 $\operatorname{EKF}(\infty)$ deinterleaver with $\mu=\infty, r_{k}^{\mu}, q_{k}^{\mu}$ are constant, and $X_{k}^{\text {diag }}=X_{k} X_{k}^{\prime}$; therefore, $R_{k}$ and $Q_{k}$ are constant.

\section{B. The State Update Equations}

The update equations for the extended Kalman filter are

$$
\begin{aligned}
\hat{x}_{k / k} & =\hat{x}_{k / k-1}+K_{k}^{\mu}\left[y_{k+1}-h_{k}\left(\hat{x}_{k / k-1}\right)\right] \\
\hat{x}_{k+1 / k} & =f_{k}\left(\hat{x}_{k / k}\right)
\end{aligned}
$$

where $\hat{x}_{k+1 / k}$ is the filtered estimate of $x_{k+1}, y_{k+1}$ is the input to the filter, $f_{k}$ and $h_{k}$ are defined in (4) and $K_{k}^{\mu}$ is defined in (12). The initialization here is provided by $\hat{x}_{0 /-1}=\hat{x}_{0}$, where $\hat{x}_{0}=\left[\hat{T}_{0}{ }^{\prime} \hat{t}_{0}\right]^{\prime}$.

For both cases 1 and 2 deinterleavers, notice that the update equations work with $f_{k}, h_{k}$ rather than the smoothed versions $f_{k}^{\mu}, h_{k}^{\mu}$. That is, there is no need to work with a model with smooth nonlinearities except for the calculation of $K_{k}^{\mu}$.

\section{SOME EXAMPLES}

In this and the following section, results obtained using computer generated pulse trains are examined. The trains have randomly generated periods and phases, with the initial pulse from each train falling within one period of time zero. Here, it is assumed that there is no noise present on the $\operatorname{EKF}(\mu)$ input signal. The effect of noise and other robustness issues are examined in Section V.

For the following discussion, the concept of the ratio of periods is needed. For any set of $M$ pulse train sources that
TABLE I

Comparison of the $\operatorname{EKF}(\mu)$ Deinterleavers With $\hat{T}_{0}$ Within $10 \%$ of $T_{0}$

\begin{tabular}{c|c|c}
\hline$R P$ & \multicolumn{2}{|c}{$\begin{array}{c}\text { Number of sources deinterleaved } \\
\text { Case 1 }\end{array}$} \\
\hline 2 & $>7$ & $>9$ \\
3 & 7 & 9 \\
4 & 6 & 9 \\
5 & 5 & 7 \\
6 & 3 & 8 \\
7 & None & 8 \\
8 & None & 7 \\
9 & None & 7 \\
10 & None & 6 \\
15 & None & 6 \\
20 & None & 6 \\
\hline
\end{tabular}

TABLE II

Comparison of the $\operatorname{EKF}(\mu)$ Deinterleavers with $\hat{T}_{0}=T_{0}$

\begin{tabular}{c|c|c}
\hline$R P$ & \multicolumn{2}{|c}{$\begin{array}{c}\text { Number of sources deinterleaved } \\
\text { Case 1 }\end{array}$} \\
\hline 2 & $>9$ & $>9$ \\
$3-9$ & 9 & 9 \\
10 & 6 & 7 \\
15 & 2 & 6 \\
20 & None & 6 \\
\hline
\end{tabular}

form the interleaved signal, the ratio of periods $(R P)$ for that signal is defined as

$$
R P=\frac{\max \left(T^{(1)}, \cdots, T^{(M)}\right)}{\min \left(T^{(1)}, \cdots, T^{(M)}\right)}
$$

where $R P$ must be greater than or equal to 1 .

\section{A. Comparison of Cases 1 and 2 EKF Deinterleavers}

As stated in Section III, for the case 1 deinterleaver $\operatorname{EKF}(\infty)$, it is assumed that $F_{k}^{\mu}$ and $H_{k}^{\mu}(8)$ have no sensitivity functions. This leads to simpler calculations. In this section, a comparison will be made between the extended Kalman filter deinterleaver using $X_{k}$ (case 1) and $X_{k}^{\mu}$ (case 2).

Tables I and II show the results obtained with different initial conditions using the cases 1 and $2 \operatorname{EKF}(\mu)$ deinterleaver. Comparison of these results show that the case $2 \operatorname{EKF}(\mu)$ deinterleaver is the better of the two. The results are similar for low $R P$, but the effectiveness of the case $1 \operatorname{EKF}(\infty)$ deinterleaver falls off rapidly as $R P$ increases beyond a threshold. For a $10 \%$ uncertainty in the periods, the case $1 \operatorname{EKF}(\infty)$ deinterleaver never works as well as the case 2 $\operatorname{EKF}(\mu)$ deinterleaver. When the periods are known exactly, the threshold is an $R P$ of 9 . With any initial conditions for the train periods, there comes a point above the threshold where the case $1 \operatorname{EKF}(\infty)$ deinterleaver ceases to work, being unable to deinterleave even a two source pulse train.

The Effect of Initial Conditions: The results shown in Table I were generated with the same initial conditions for both $\operatorname{EKF}(\mu)$ deinterleavers. It is assumed that the periods of the trains are known to within $10 \%$ (e.g., by order $N \log N$ spectral studies as in [5]) and that the first pulse in each train lies within one period of time zero. The initialization for the 
error covariance reflects this.

$$
P_{0}=\left[\begin{array}{cc}
\left(\hat{T}_{0}^{(i)}\right)^{2} / 100 & 0 \\
0 & \left(\hat{T}_{0}^{(i)}\right)^{2} / 10
\end{array}\right] .
$$

Even with these constraints on the initial conditions for period and phase, it is found that both cases 1 and 2 deinterleavers are sensitive to the initial conditions chosen. For this reason, a bank of ten deinterleavers is considered with random phases, and the one leading to the least average prediction error squared is selected. For this deinterleaving method to retain its computational advantage over other methods of order $N^{2}$, the number of filters in the bank should be much less than $N$.

As can be seen from Tables I and II, it becomes increasingly difficult to deinterleave the signals as the number of sources or $R P$ increase. This is because the set of possible initial phases increase rapidly with the number of sources $M$ as well as with $R P$ since phase has been defined as being linked to period. As this set increases, it contains more local minima that the $\operatorname{EKF}(\mu)$ deinterleavers tend toward but does not lead to the correct deinterleaving of the pulse trains. Therefore, as the number of pulse trains increases, the choice of initial phase becomes more important. However, the $\operatorname{EKF}(\mu)$ deinterleavers are not only convergent locally in the phase space; therefore, regardless of the number of sources present or the value of $R P$, the initial estimate for $\hat{t}_{0}$ does not necessarily need to be close to $t_{0}$. Therefore, the assumption that $t_{0}$ falls within one period of time zero that is made for choosing $\hat{t}_{0}$ does not need to be correct for the pulse trains to be deinterleaved.

When no a priori knowledge of the pulse train periods is assumed, the set of possible initial conditions that a filter bank must scan becomes much larger. A random generation of periods could be employed; however, the $\operatorname{EKF}(\mu)$ deinterleavers are effective (convergent) only locally in the period space. Table I therefore shows the performance of both deinterleavers when there is no knowledge of the periods, assuming that there can be up to $N$ randomized deinterleavers used.

\section{B. The Case $2 \operatorname{EKF}(\mu)$ Deinterleaver}

As the case $2 \operatorname{EKF}(\mu)$ deinterleaver is the best of the two presented, we will concentrate on it for the rest of the paper. Figs. 3 and 4 show the successful deinterleaving of pulse trains by this deinterleaver. Fig. 3 is an example of period estimation with a two-source input. It should be noted that the $\operatorname{EKF}(\mu)$ takes longer to lock onto the pulse train with the highest period. This is due to less pulses from this train being present in the input signal.

Fig. 4 is an example of period estimation with an eightsource input.

\section{RoBUSTNESS ISSUES}

In this section, only the results from the case $2 \operatorname{EKF}(\mu)$ deinterleaver are examined.

\section{A. Jitter Noise}

Noise is present in all real-world situations, so for this method of pulse train deinterleaving to be useful, it must be robust to the effects of noise. Such noise is included in our

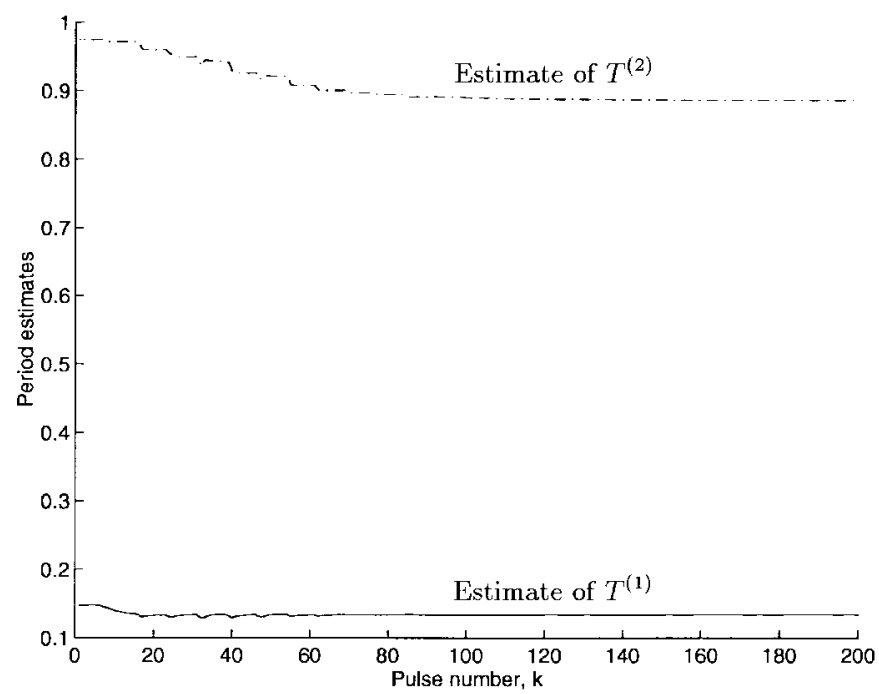

Parameters: $T_{0}^{\prime}=[0.1340,0.8858], t_{0}^{\prime}=[0.0701,0.1692]$ Initial estimates: $\hat{T}_{0}^{\prime}=[0.1474,0.9744], \hat{t}_{0}^{\prime}=[0.0137,0.1283]$

Fig. 3. Evolution of periods, case $2 \operatorname{EKF}(\mu)$ deinterleaver, two source input.

signal model. Table III shows how different levels of noise effect the case 2 deinterleaver with known pulse train periods. The error given is the percentage of pulses assigned to an incorrect train after a lock on the pulse train periods has been achieved. The maximum number of sources successfully deinterleaved is also given in each case.

As the noise increases, the number of sources successfully deinterleaved decreases, and the number of errors increases. It should be noted that in a set of pulse trains, the $\operatorname{EKF}(\mu)$ deinterleaver makes the most errors in the estimate of the train with the lowest period. This is because the pulses in this train come closest together and are therefore more easily disrupted by noise. In addition, when the periods of two trains are similar, noise can cause pulse train skipping; the pulse train estimates swap pulse train sources. This occurs when the periods differ by approximately the noise variance.

\section{B. Missing Pulses}

Table IV shows the effect of a percentage of pulses being removed from the input to the $\operatorname{EKF}(\mu)$ deinterleaver. Of course, such missing pulses were not incorporated into the signal model so there is no a priori expectation of such by the $\operatorname{EKF}(\mu)$. The same trains are used as in Section V-A. Here, there is no noise present on the EKF input, and each pulse in the input is given a 1,5 , or $10 \%$ probability of not being present.

The $\operatorname{EKF}(\mu)$ deinterleaver is not robust to missing pulses. A dropoff in effectiveness is apparent with even $1 \%$ of pulses missing. It is also possible for the $\operatorname{EKF}(\mu)$ deinterleaver to lose its lock on the pulse trains once it has been established. This occurs if there are a lot of pulses in the same region that are missing. With no missing pulses, this phenomenon is only observed under high noise conditions.

To cope with missing pulses, clearly, a modification of the present algorithm, such as in [4], is necessary. There, a comparison of the prediction errors is made, both assuming 


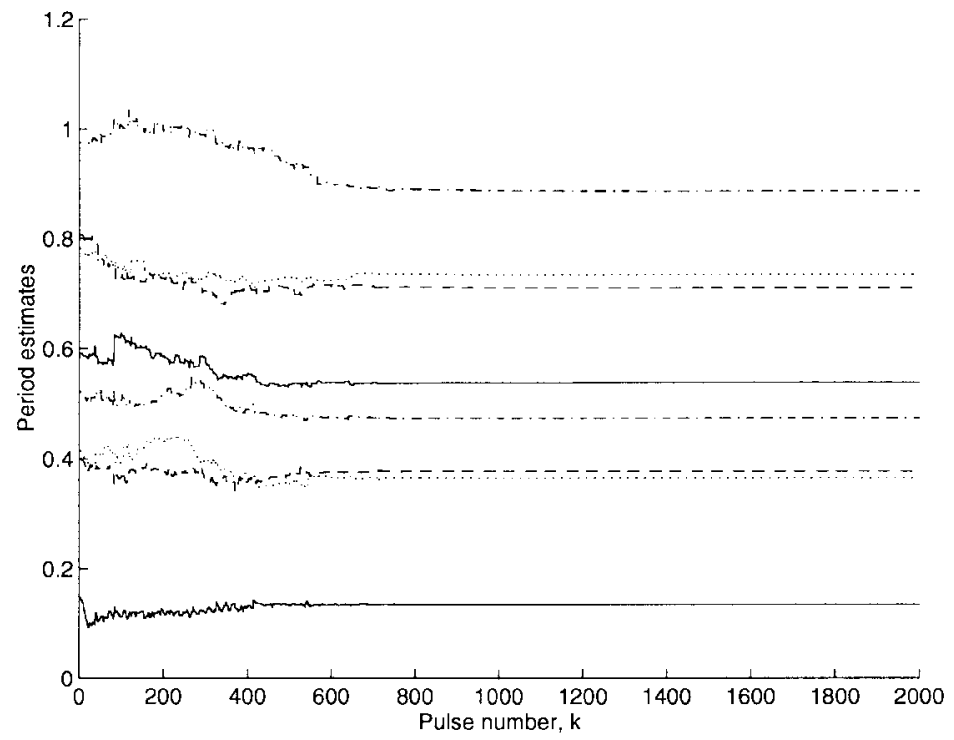

Parameters: ' $I_{0}^{\prime \prime}=[0.1340,0.3644,0.3769,0.4736,0.5377,0.7099,0.7332,0.8858]$,

$t_{0}^{\prime}=[0.0706,0.0159,0.3198,0.4201,0.0677,0.1255,0.2343,0.0773]$

Initial estimates:

$\hat{T}_{0}^{\prime}=[0.1474,0.4009,0.4146,0.5210,0.5915,0.7809,0.8065,0.9744]$,

$\hat{t}_{0}^{\prime}=[0.1356,0.3467,0.1769,0.1337,0.1930,0.3089,0.1706,0.7894]$

Fig. 4. Evolution of periods, case $2 \operatorname{EKF}(\mu)$ deinterleaver, eight source input.

TABLE III

EfFect of Noise on the CASE $2 \operatorname{EKF}(\mu)$ Deinterleaver

\begin{tabular}{c|c|c}
\hline $\begin{array}{c}\text { Noise } \\
\sigma_{w}^{2}\end{array}$ & \multicolumn{2}{|c}{$\begin{array}{c}\text { Number of sources (\% error) } \\
\text { RP3 }\end{array}$} \\
\hline 0.001 & $8(1.5 \%)$ & $7(2.1 \%)$ \\
0.01 & $8(9.6 \%)$ & $7(12.5 \%)$ \\
0.02 & $7(14.6 \%)$ & $6(18.2 \%)$ \\
\hline
\end{tabular}

TABLE IV

Effect of Missing Pulses on the Case 2 EKF $(\mu)$ Deinterleaver

\begin{tabular}{c|c|c}
\hline Missing pulses & \multicolumn{3}{|c}{$\begin{array}{c}\text { Number of sources } \\
\text { RP3 }\end{array}$} & RP7 \\
\hline $1 \%$ & 6 & 6 \\
$5 \%$ & 2 & 3 \\
$10 \%$ & 2 & 2 \\
\hline
\end{tabular}

that there is no missing pulse and assuming there is. Such an approach adapted to the $\operatorname{EKF}(\mu)$ setting is beyond the scope of this paper.

\section{CONCLUSION}

The most important aspect of the method for pulse train deinterleaving presented here is its computational efficiency. The use of an extended Kalman filter allows computations of order $N$, rather than $N^{2}$, which is typical for other deinterleaving methods: $N$ is the number of pulses to be processed. It is advantageous to use information about the pulse train periods that can be obtained using computations of order $N \log N$ [5]. The $\operatorname{EKF}(\mu)$ deinterleaving method can therefore use a bank of much less than $N$ deinterleavers with different initial conditions and still be more efficient than other methods. This decreases the sensitivity to initial conditions observed in a single $\operatorname{EKF}(\mu)$ deinterleaver.

The $\operatorname{EKF}(\mu)$ deinterleaver is robust to noisy pulse time of arrival data but not to missing pulses. It is possible that a modification to this method needing more computational effort could improve its response when missing pulses are present.

\section{REFERENCES}

[1] R. G. Wiley, Electronic Intelligence: The Analysis of Radar Signals. Norwood, MA: Artech House, 1993.

[2] H. K. Mardia, "New techniques for the deinterleaving of repetitive sequences," Proc. Inst. Elect. Eng. F, vol. 136, pp. 149-154, 1989.

[3] D. J. Milojević and B. M. Popović, "Improved algorithm for the deinterleaving of radar pulses," Proc. Inst. Elect. Eng. F vol. 139, pp. 98-104, 1992.

[4] J. B. Moore and V. Krishnamurthy, "Deinterleaving pulse trains using discrete-time stochastic dynamic-linear models," IEEE Trans. Signal Processing, vol. 42, pp. 3092-3103, Nov. 1994.

[5] R. J. Orsi, J. B. Moore, and R. E. Mahony, "Spectrum estimation of interleaved pulse trains," in Proc. ISSPA, Gold Coast, Australia, 1996, pp. $125-128$, vol. 2.

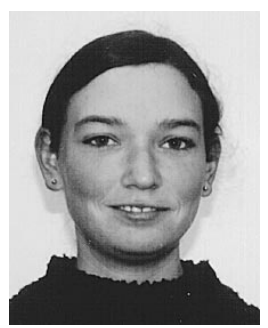

Tanya Conroy received the B.S. degrees in science and engineering in 1993 and 1995, respectively, both from the Australian National University, Canberra. She is currently studying for the Ph.D. degree in systems engineering at the Australian National University.

Her research interests include signal processing and robotic vision systems. 


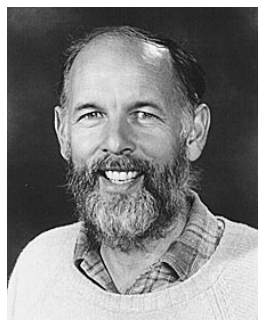

John B. Moore (F'79) was born in China in 1941. He received the B.S. and M.S. degrees in electrical engineering in 1963 and 1964, respectively, and the doctorate degree in electrical engineering from the University of Santa Clara, Santa Clara, CA, in 1967.

He was appointed Senior Lecturer with the Electrical Engineering Department, University of Newcastle, Newcastle, Australia, in 1967 and promoted to Associate Professor in 1968 and Full Professor (personal chair) in 1973. He was Department Head from 1975 to 1979 . In 1982, he was appointed Professorial Fellow in the Department of Systems Engineering, Research School of Physical Sciences, Australian National University, Canberra, and promoted to Professor in 1990. He has been Head of the Department since 1992 and is now with the Research School of Information Sciences and Engineering. He has held visiting academic appointments at the University of Santa Clara in 1968; the University of Maryland, College Park, in 1970, Colorado State University, Fort Collins, and Imperial College, London, U.K., in 1974; the University of California, Davis in 1977; the University of Washington, Seattle, in 1981; Cambridge University, Cambridge, U.K., and the National University of Singapore in 1985; the University of California, Berkeley, in 1987, 1989, and 1991; the University of Alberta, Edmonton, in 1992, 1993, and 1994; University of Regensburg, Regensburg, Germany, in 1993; Toshiba Chair, Institute of Industrial Science, University of Tokyo, Tokyo, Japan, in September 1993; the University of Maryland, again, in 1994; the Imperial College of Science, Technology, and Medicine, London, U.K., in 1995; the Technical University of Munich, Munich, Germany, in 1995; University of Würzberg, Würzberg, Germany, in 1996; the Chinese University of Hong Kong and the University of Würzberg in 1997. He has spent periods in industry as a Design Engineer and as a Consultant and, currently, has research grants from industry and government laboratories. He is a Team Leader in the Cooperative Research Centre for robust and adaptive systems in the Department. His current research is in control and communication systems and signal processing. He is coauthor, with B. D. O. Anderson of three books: Linear Optimal Control (Englewood Cliffs, NJ: Prentice-Hall, 1971), Optimal Filtering (Englewood Cliffs, NJ: Prentice-Hall, 1979), and Optimal ControlLinear Quadratic Methods (Englewood Cliffs, NJ: Prentice-Hall, 1989). He is coauthor of a book, with U. Helmke, entitled Optimization and Dynamical Systems (New York: Springer-Verlag, 1993). He cowrote the book with R. Elliott and L. Aggoun entitled Hidden Markov Model Estimation and Control via Reference Methods (New York: Springer-Verlag, 1995) and the book, with T. T. Tay and I. Mareels, entitled High Performance Control (Boston, MA: Birkhäsuer, 1997).

Dr. Moore a Fellow of the both Australian Academy of Technological Sciences and Engineering and the Australian Academy of Science. 